\title{
$\mathrm{R} C \& \mathrm{C}$
}

REVISTA DE CONTABILIDADE E CONTROLADORIA

\section{UNIVERSIDADE FEDERAL DO PARANÁ - UFPR}

Periódico quadrimestral, digital e gratuito, do Programa de Pós-Graduação em Contabilidade do Departamento de Contabilidade - Setor de Ciências Sociais Aplicadas.

\section{5, N. 2, MAIO/AGO. 2013 \\ DATA DA PUBLICAÇÃO: 15 DE SETEMBRO DE 2013}

CORPO DIRETIVO - DIRECTIVE BOARD

Zaki Akel Sobrinho, Reitor da Universidade Federal do Paraná, Brasil Vicente Pacheco, Diretor do Setor de Ciências Sociais Aplicadas, UFPR Jackson Ciro Sandrini, Chefe do Departamento de Contabilidade, UFPR Márcia M. Bortolocci Espejo, Coord. do Programa de Pós- Graduação em Contabilidade, UFPR Luciano Márcio Scherer, Coordenador do Curso de Ciências Contábeis, UFPR

EDITOR - EDITOR

Jorge Eduardo Scarpin, Departamento de Contabilidade, UFPR

REVISÃO DE NORMALIZAÇÃO - REVISION OF STANDARDS

Daiana Bragueto Martins, Universidade Federal do Paraná, UFPR

REVISÃO DE LÍNGUA PORTUGUESA - REVISION OF PORTUGUESE LANGUAGE

Daiana Bragueto Martins, Universidade Federal do Paraná, UFPR

\section{REVISÃO DE LÍNGUA INGLESA - REVIEW OF ENGLISH} LANGUAGE

Ademir Clemente, Departamento de Contabilidade, UFPR 


\section{COMITÊ DE POLIITICA EDITORIAL - EDITORIAL BOARD}

Ademir Clemente, Departamento de Contabilidade, UFPR

Márcia Maria Bortolocci Espejo, Departamento de Contabilidade, UFPR

Romualdo Douglas Colauto, Departamento de Contabilidade, UFPR

\section{CORPO EDITORIAL - EDITORIAL BOARD}

Ana Paula Mussi Szabo Cherobim, Universidade Federal do Paraná - PR, Brasil Antonio Carlos Dias Coelho, Universidade Federal do Ceará - CE, Brasil

Aracéli Cristina de S. Ferreira, Universidade Federal do Rio de Janeiro - RJ, Brasil

Cláudio Parisi, Fundação Escola de Comércio Álvares Penteado - SP, Brasil

Edgard Bruno Cornachione Junior, Universidade de São Paulo - SP, Brasil

Ernani Ott, Universidade do Vale do Rio dos Sinos - RS, Brasil

Fábio Frezatti, Universidade de São Paulo, SP, Brasil

Ilse Maria Beuren, Universidade Regional de Blumenau - SC, Brasil

Jacqueline V.Alves. da Cunha, Universidade Federal de Minas Gerais - MG, Brasil

Lauro Brito de Almeida, Universidade Federal do Paraná - PR, Brasil

José Alonso Borba, Universidade Federal de Santa Catarina - SC, Brasil

Luiz Carlos Miranda, Universidade Federal de Pernambuco - PE, Brasil

Márcia Martins Mendes De Luca, Universidade Federal do Ceará - CE, Brasil

Maísa de Souza Ribeiro, Universidade de São Paulo - SP, Brasil

Marcos Antonio de Souza, Universidade do Vale do Rio dos Sinos - RS, Brasil

Masayuki Nakagawa, Universidade de São Paulo - SP, Brasil

Paulo Roberto Barbosa Lustosa, Universidade Federal de Brasília - DF, Brasil

Rodrigo Oliveira Soares, Universidade Federal do Paraná - PR, Brasil

Valmor Slomski, Universidade de São Paulo - SP, Brasil

\section{EDITORES SISTEMA SER}

Paulo Ugolini

\section{BIBLIOTECÁRIA}

Moema Bassfeld

\section{(C) UFPR - Universidade Federal do Paraná}

A Política Editorial da Revista é a do acesso livre e gratuito na disseminação, recuperação da informação e visibilidade científica. Para tanto, todos os artigos publicados podem ser baixados integral e gratuitamente. 
A operacionalização e acesso à revista é possibilitado pelo Sistema Eletrônico de Revistas - SER, da PRPPG-UFPR, vinculado ao Sistema Eletrônico de Editoração de Revistas (Open Journal Systems - OJS), desenvolvido pelo Public Knowledge Project PKP.

Disponível em: http://www.ser.ufpr.br/rcc

Revista de contabilidade e controladoria / Universidade Federal do Paraná, Programa de Pós graduação Mestrado em Contabilidade. - v. 1, n. 1 (2009)-. Curitiba : UFPR, 2009-

v.

Quadrimestral

ISSN: $1984-6266$

1. Contabilidade - Periódicos. I. Universidade Federal do Paraná, Programa de Pós-Graduação Mestrado em Contabilidade

CDD- 657

\section{CATALOGAÇÃO NA FONTE UFPR - SISTEMA DE BIBLIOTECAS - SIBI DIRETÓRIOS DE REGISTRO DE PERIÓRIDOS}

Open Journal Systems - OJS

LATINDEX - Universidad Nacional Autónoma de México (UNAM)

\section{Endereço:}

Universidade Federal do Paraná - PPG Mestrado em Contabilidade

Av. Pref. Lothário Meissner, 632 Campus III, Jd Botânico CEP 80210-170 Curitiba - Paraná - Brasil

Telefone: 5541 3360-4411

Fax: $55413360-4193$

E-mail: rccontroladoria@ufpr.br 